\title{
EXTINCTION MAPS OF DUST CLOUDS FROM SURFACE PHOTOMETRY
}

\author{
K. J. Seidensticker and Th. Schmidt-Kaler \\ Astronomical Institute \\ Ruhr-Universität Bochum \\ Postfach 102148 \\ D-4630 Bochum 1 FRG
}

\begin{abstract}
We present a new method, based on surface photometry, which allows one to make the spatial resolution of extinction maps of dark clouds as fine as necessary. Applying this technique to the Coalsack $\left(l=302^{\circ}, b=0^{\circ}\right)$, we derived an extinction map and the dust mass $M_{\mathrm{D}}=(62 \pm 25) M_{\odot}$. The density was found to vary $\rho \sim r^{-0.8}$, typical for stable clouds.
\end{abstract}

\section{INTRODUCTION}

Usually, extinction maps of dust clouds are obtained by comparing star numbers in small areas with those of neighboring, supposedly dust-free, regions. Surface photometry offers a new technique. Here one measures the combined contributions of the direct light of the stars and of the light scattered by dust, i.e. the diffuse galactic light (DGL).

This new method has two advantages: (1) the resolution can be made as fine as necessary; and (2) the background variation can be better approximated than in the case of star counts. On the other hand, one has to model the DGL assuming the albedo $a$ and the asymmetry factor $g$ of the dust particles. But these two scattering parameters can be reasonably well determined by combining star counts with surface photometry.

\section{METHOD AND DATA}

We applied the new technique to the Coalsack, since (1) the scattering model of Mattila (1970, 1976 ) is best suited to dust clouds at the galactic equator; and (2) we had determined the surface brightnesses in U, B, V, R (Seidensticker et al. 1982).

Since the background intensity $I_{\mathrm{B}}(\lambda)$ is diminished by the extinction $A(\lambda)$, but increased by the scattered light $I_{\mathrm{S}}(\lambda)$ and the contribution of foreground stars $I_{\mathrm{F}}(\lambda)$, the intensity of a pixel $I(\lambda)$ can be described by $I(\lambda)=I_{\mathrm{B}}(\lambda) \cdot 10^{(-0.4 A(\lambda))}+I_{\mathrm{S}}(\lambda)+I_{\mathrm{F}}(\lambda)$.

For each spectral range, we fitted the brightness variation in $b$ at three different longitudes as well as the variation in $l$, in order to calculate $I_{\mathrm{B}}$ for each pixel within the Coalsack.

Mattila $(1970,1976)$ computed the ratio of incoming to scattered light as a function of optical thickness $\tau$, albedo $a$ and asymmetry factor $g$. We approximated his tables by an empirical function up to $\tau=3$ with a maximum deviation of $2 \%$.

Comparing our surface photometry in B with the star counts within selected areas we determined the brightness of the DGL and its variation with $b$ for two galactic longitudes. Applying a DGL-model of Lillie and Witt (1976) the dust parameters $a=0.65 \pm 0.10$ and $g=$ $0.75 \pm 0.10$ were found. 


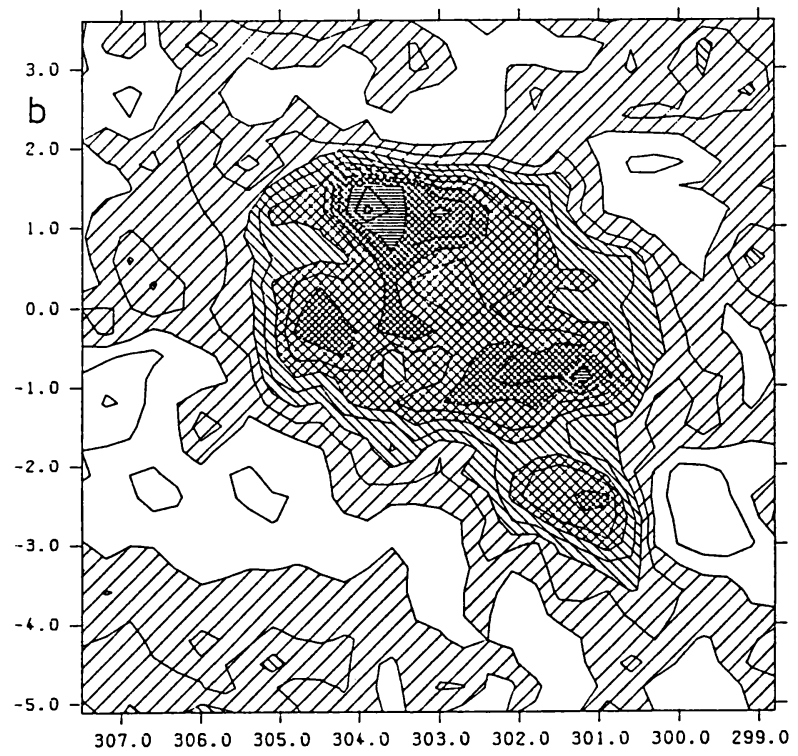

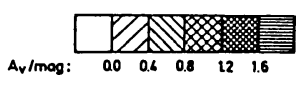

Figure 1. Extinction map $A_{\mathrm{v}}(l, b)$ of the Coalsack. The resolution is $0.3^{\circ} \times 0.3^{\circ}$, the extinction contours differ by 0.2 .

\section{RESULTS}

Independent extinction maps for all four spectral regions were iteratively computed: starting with a minimum extinction $\left(I_{S}(\lambda)=0\right.$ in equation [1]), then calculating $I_{S}(\lambda)$ and determining a new $A(\lambda)$ from equation (1). The difference $\left|A(\lambda)_{\text {old }}-A(\lambda)_{\text {new }}\right|$ was usually $\leq 0$. 02 after two iterations. Mean ratios $f_{\lambda}=\Sigma A_{\lambda} / \Sigma A_{\mathrm{V}}$ were determined and the $A_{\mathrm{R}}, A_{\mathrm{B}}$, and $A_{\mathrm{U}}$ maps converted to $A_{\mathrm{V}}$ and averaged with the $A_{\mathrm{V}}$ extinction map (Figure 1).

\section{DISCUSSION}

The dust mass $M_{\mathrm{D}}$ of a dust cloud (in $M_{\odot}$ ) can be expressed by $M_{\mathrm{D}}=0.18 \cdot \sigma d^{2} \cdot a \rho / Q_{\mathrm{V}}$ - $\Sigma A_{\mathrm{V}, \mathrm{i}}$, where $d$ is the distance in pc, $\sigma$ the solid angle of a pixel in square degrees, $a \rho / Q_{\mathrm{V}}$ describes the material properties of the dust particles and $\Sigma A_{\mathrm{V}, \mathrm{i}}$ the total extinction. With $d=$ $(210 \pm 20) \mathrm{pc}, a \rho / Q_{\mathrm{V}}=(3 \pm 1) \times 10^{-4} \mathrm{~kg} \mathrm{~m}^{7-2}, \Sigma A_{\mathrm{V}, \mathrm{i}}=290 \pm 30\left(0 \mathrm{~m}_{3}\right.$ contour in Figure 1) we got $M_{\mathrm{D}}=(62 \pm 25) M_{\odot}$.

Cernicharo et al. (1985) described the density profile of dust clouds by $n(r, \alpha)=c \cdot r^{-\alpha}$. Comparison of our data with Cernicharo's models gives $\alpha \approx 0.8$. Falgarone and Puget (1985) found that the density structure of stable, self-gravitating dust clouds has to vary with $\alpha<1$ in the outer regions. Thus $\alpha \approx 0.8$ is evidence that the Coalsack is stable and that gravity is balanced by other forces.

\section{REFERENCES}

Cernicharo, J., Bachiller, R., and Duvert, G. 1985, Astron. Astrophys., 149, 273.

Falgarone, E. and Puget, J. L. 1985, Astron. Astrophys., 142, 157.

Lillie, C. F. and Witt, A. N. 1976, Ap. J., 208, 64.

Mattila, K. 1970, Astron. Astrophys., 9, 53.

Mattila, K. 1976, Astron. Astrophys., 47, 77.

Seidensticker, K. J., Schmidt-Kaler, Th., and Schlosser, W. 1982, Astron. Astrophys., 114, 60. 